\title{
Composition- and magnetic field-driven antiferromagnetic-weak ferromagnetic transition in $\mathrm{Bi}_{1-\mathrm{x}} \mathrm{Ca}_{\mathrm{x}} \mathrm{Fe}_{1-\mathrm{x}} \mathrm{Ti}_{\mathrm{x}} \mathrm{O}_{3}$ multiferroics
}

\author{
V. A. Khomchenko and J. A. Paixão
}

CFisUC, Department of Physics, University of Coimbra, P-3004-516 Coimbra, Portugal

The investigation aims at assessing the influence of $\mathrm{Ca}^{2+} / \mathrm{Ti}^{4+}$ co-doping on the crystal structure and magnetic properties of the $\mathrm{BiFeO}_{3}$ multiferroic exhibiting cycloidal spin ordering in the polar phase. We show that increasing the content of the substituents results in reducing the stability range of the cycloidal antiferromagnetic structure in external magnetic field. Above the critical concentration corresponding to the composition with $x=0.15$, the cycloidal order is removed to enable the formation of a weak ferromagnetic state. The spontaneous magnetization characteristic of the doping-stabilized weak ferromagnetic phase is very close to the locked magnetization releasing upon the magnetic field-induced cycloidal ordering $\leftrightarrow$ canted ordering transition observed in the compounds with $x \leq 0.15$. Since the doping-driven magnetic transformation is not accompanied by changes in the symmetry of the crystal lattice, the $\mathrm{Ca}^{2+} / \mathrm{Ti}^{4+}$ co-substitution provides a promising opportunity to combine switchable magnetization and polarization in a single phase.

Keywords: $\mathrm{BiFeO}_{3}$, Crystal structure, Magnetic materials, Multiferroics, Spin-cycloid instability

Corresponding author: Dr. V. A. Khomchenko

CFisUC, Department of Physics, University of Coimbra, P-3004-516 Coimbra, Portugal

E-mail: uladzimir@ fis.uc.pt

Phone: +351239410637

Fax: +351239829158

(C) 2016. This manuscript version is made available under the Elsevier user license http://www.elsevier.com/open-access/userlicense/1.0/ 


\section{Introduction}

Multiferroics exhibiting magnetic and electric dipole ordering in the same phase are nowadays considered as having tremendous potential for application in spin-based electronics [1]. Increasing attention is currently paid to perovskite-type $\left(\mathrm{ABO}_{3}\right)$ bismuth ferrite $\left(\mathrm{BiFeO}_{3}\right)$ [2] which remains an extremely rare example of a single-phase material demonstrating magnetoelectric coupling at room temperature. In the polar phase $\left(T<T_{\mathrm{C}} \approx 1100 \mathrm{~K} ; T_{\mathrm{C}}\right.$ denotes the ferroelectric Curie point), $\mathrm{BiFeO}_{3}$ has a rhombohedrally distorted crystal structure with the space group $R 3 c$ [3]. Below $T_{\mathrm{N}} \approx 640 \mathrm{~K}$ ( $T_{\mathrm{N}}$ stands for the Néel temperature), the compound orders antiferromagnetically (the local arrangement is of $G$-type) [4]. The first-principles calculations of the magnetocrystalline anisotropy in $\mathrm{BiFeO}_{3}$ predict that the magnetic moments of $\mathrm{Fe}^{3+}$ should be oriented perpendicular to the threefold axis (this orientation permits a canting of the antiferromagnetic (AFM) sublattices resulting in the appearance of a small macroscopic magnetization) [5]. Magnetoelectric coupling, however, affects the magnetic ordering and stabilizes a long-period cycloid structure incompatible with the existence of weak ferromagnetism [6]. Application of a strong enough (H 200 kOe) magnetic field suppresses the cycloidal modulation, thus giving rise to the formation of a spatially-homogeneous canted magnetic structure with $M \approx 0.25 \mathrm{emu} / \mathrm{g}$ [7]. Although isovalent replacement of $\mathrm{Bi}^{3+}$ with $\mathrm{Ln}^{3+}$ ( $\mathrm{Ln}=$ lanthanide) is proven to reduce the threshold field [8, 9], it seems to be unable to stabilize the weak ferromagnetic (wFM) polar state at $H=0$ [10-12]. Isovalent substitution of $\mathrm{Fe}^{3+}$ with $\mathrm{Sc}^{3+}[13,14]$ or $\mathrm{Mn}^{3+}[15,16]$ does not change the magnetic state characteristic of $\mathrm{BiFeO}_{3}$. Aliovalent doping, in contrast, can effectively influence the magnetic behavior to induce the AFM-wFM transformation without affecting the crystal symmetry. Indeed, the spontaneous magnetization associated with the $R 3 c$ phase has been found in $\mathrm{Ca}^{2+}$ [17], $\mathrm{Sr}^{2+}$ [18], $\mathrm{Ti}^{4+}$ [19], $\mathrm{Zr}^{4+}$ [20], and $\mathrm{Nb}^{5+}$-doped [21] $\mathrm{BiFeO}_{3}$-based perovskites. Discovery of a correlation between the magnetic and morphological evolution in the acceptor- and donor-doped series implies that the origin of doping-driven instability of the cycloidal antiferromagnetic order should be closely connected with the mechanism of charge compensation involving the formation of defects (anion and cation vacancies, respectively) in the crystal lattice of the aliovalent-doped materials [21-24]. It is important to note that the spontaneous magnetization has also been found in the polar compounds of the $\mathrm{Bi}_{1-x}^{3+} \mathrm{Ca}_{x}^{2+} \mathrm{Fe}_{1-x}^{3+} \mathrm{Ti}_{x}^{4+} \mathrm{O}_{3}^{2-}$ system [2527], in which the appearance of weak ferromagnetism can hardly be associated with the substitutioninduced changes in the defect structure. While the previous investigations of the $\mathrm{Bi}_{1-\mathrm{x}} \mathrm{Ca}_{\mathrm{x}} \mathrm{Fe}_{1-\mathrm{x}} \mathrm{Ti}_{\mathrm{x}} \mathrm{O}_{3}$ series have shown that the lightly-doped $(x \sim 0.05)$ samples exhibit a mixed AFM-wFM behavior with a 
relatively large spontaneous magnetization (up to $\sim 0.1 \mathrm{emu} / \mathrm{g}$ ) [25, 27], more recent magnetic measurements of the $\mathrm{Bi}_{0.95} \mathrm{Ca}_{0.05} \mathrm{Fe}_{0.95} \mathrm{Ti}_{0.05} \mathrm{O}_{3}$ compound have not revealed the presence of ferromagnetic contribution [23, 24]. To verify the pioneering data [25-27] and examine whether the chargecompensatory $\mathrm{Ca}^{2+} / \mathrm{Ti}^{4+}$ co-doping can really act to stabilize the weak ferromagnetic polar state, solidstate synthesis and study of the crystal structure, ferroelectric domain structure, and magnetic properties of the $\mathrm{Bi}_{1-\mathrm{x}} \mathrm{Ca}_{\mathrm{x}} \mathrm{Fe}_{1-\mathrm{x}} \mathrm{Ti}_{\mathrm{x}} \mathrm{O}_{3}(x \leq 0.02)$ perovskites have been carried out.

\section{Experimental}

Polycrystalline samples of $\mathrm{Bi}_{1-\mathrm{x}} \mathrm{Ca}_{\mathrm{x}} \mathrm{Fe}_{1-\mathrm{x}} \mathrm{Ti}_{\mathrm{x}} \mathrm{O}_{3}(x=0.05,0.1,0.15,0.2)$ were prepared by solid state reaction between $\geq 99 \%$ pure $\mathrm{Bi}_{2} \mathrm{O}_{3}, \mathrm{CaCO}_{3}, \mathrm{Fe}_{2} \mathrm{O}_{3}$ and $\mathrm{TiO}_{2}$ powders $(<10 \mu \mathrm{m}$, Sigma-Aldrich) taken in stoichiometric amounts. The reagents were mixed for $1 \mathrm{~h}$ using an agate mortar and pestle. The mixtures were pressed into pellets of $10 \mathrm{~mm}$ diameter and 2-3 $\mathrm{mm}$ thickness and annealed in air at $900^{\circ} \mathrm{C}$ $(x=0.05), 920^{\circ} \mathrm{C}(x=0.1), 940^{\circ} \mathrm{C}(x=0.15)$ or $960^{\circ} \mathrm{C}(x=0.2)$ for $30 \mathrm{~h}$ (annealing temperature was increased with increasing $\mathrm{Ca} / \mathrm{Ti}$ content to ensure the formation of chemically homogeneous solid solutions). Phase and structure analysis was performed on crushed pellets using a Bruker D8 Advance diffractometer with $\mathrm{Cu} K_{\alpha}$ radiation. The X-ray diffraction (XRD) patterns were collected by step scanning in the angle range $15^{\circ} \leq 2 \theta \leq 100^{\circ}$ with a step of $0.01^{\circ}$ and a dwell of $2 \mathrm{~s}$ per step. The diffraction data were analyzed by the Le Bail method [28] using the FullProf software package [29]. Morphology and local ferroelectric properties of ceramics were investigated with a NTEGRA Prima (NT-MDT) scanning probe microscope operating in atomic force microscopy and piezoresponse force microscopy (PFM) modes (the NSG30 probes were used). Images of ferroelectric domains were recorded under an applied $A C$ voltage with an amplitude $V_{\mathrm{AC}}=5 \mathrm{~V}$ and a frequency $f=100 \mathrm{kHz}$. Magnetic measurements of samples were performed with a cryogen-free Physical Properties Measurement System (PPMS DynaCool, Quantum Design).

\section{Results and Discussion}

Analysis of the XRD patterns obtained for the $\mathrm{Bi}_{1-\mathrm{x}} \mathrm{Ca}_{\mathrm{x}} \mathrm{Fe}_{1-\mathrm{x}} \mathrm{Ti}_{\mathrm{x}} \mathrm{O}_{3}(x=0.05,0.1,0.15,0.2)$ samples at room temperature suggests that the doped compounds retain the crystal symmetry characteristic of the pure $\mathrm{BiFeO}_{3}$ [3]. Indeed, indexing the diffraction peaks performed using the hexagonal cell with $a=\sqrt{2} a_{p}$ and $c=2 \sqrt{3} c_{p} \quad\left(a_{p} \approx c_{p} \sim 4 \AA\right.$ are the parameters of the primitive 
pseudo-cubic perovskite subcell) has yielded the systematic extinctions of reflections consistent with the space group $R 3 c$. In contrast to the results of Kumar and Kar [27], no additional peaks indicative of the presence of impurities or secondary perovskite phase has been found. The results of the structural investigation are consistent with the earlier observations of Karpinsky et al [25] and Wang et al [26] suggesting no symmetry changes within the concentration range $x \leq 0.2$. An example of the successful fitting of the XRD data carried out using a single-phase ' $R 3 c^{\prime}$ model is shown in Fig. 1 . Increasing the content of substituting elements gives rise to a contraction of the unit cell, as can be followed from a shift of the diffraction peaks towards larger $2 \theta$ values (the cental inset of Fig. 1). The contraction associated with a change in the average size of the ions populating the $A$ - and $B$-sites $\left(r_{\mathrm{Ca}^{2+}}<r_{\mathrm{Bi}^{3+}}, r_{\mathrm{Ti}^{4+}}<r_{\mathrm{Fe}^{3+}}\right)[30]$ is anisotropic and occurs mainly along the polar $c$ axis (the right inset in Fig. 1). Such a behavior is consistent with the structural evolution characteristic of Ti-doped bismuth ferrites [22, 31] and implies the decrease of ferroelectric polarization with increasing $\mathrm{Ca} / \mathrm{Ti}$ concentration.

In contrast to the results of the previous investigations [25-27], our magnetic measurements of the $\mathrm{Bi}_{1-\mathrm{x}} \mathrm{Ca}_{\mathrm{x}} \mathrm{Fe}_{1-\mathrm{x}} \mathrm{Ti}_{\mathrm{x}} \mathrm{O}_{3}(x \leq 0.15)$ compounds have not revealed any obvious contribution associated with the existence of a doping-stabilized wFM phase (Fig. 2). The low-doped samples exhibit a metamagnetic behavior specific to the field-induced transformation of the cycloidal magnetic structure [8]. The threshold field triggering the onset of the cycloidal order $\leftrightarrow$ canted order transition decreases with increasing $\mathrm{Ca} / \mathrm{Ti}$ content. In the $x=0.05$ compound, the deviation from a linear $M(H)$ dependence starts to be observed at $H \sim 50 \mathrm{kOe}$. The nonlinear increase of the magnetization continues up to the highest fields available in the experiment. The $M(H)$ curve obtained for the $x=0.1$ sample has two linear parts (at $H<25$ kOe and $H>65 \mathrm{kOe}$ ) which mark the stability ranges of the cycloidal and the canted phase, respectively. In the $x=0.15$ compound, the application of a magnetic field results in immediate transformation of the cycloidal structure, as can be seen from the nonlinear increase of the magnetization taking place in the low-field range. The canted magnetic order is fully stabilized at $H>35 \mathrm{kOe}$. The transformation of the magnetic structure is not irreversible: decreasing the field results in a drop of the magnetization, so the remanent magnetization of the sample remains very small $\left(M_{\mathrm{r}} \approx 0.035 \mathrm{emu} / \mathrm{g}\right)$. Further increase of the $\mathrm{Ca} / \mathrm{Ti}$ concentration stabilizes a weak ferromagnetic state. Indeed, the $x=0.2$ sample exhibits the field dependence of magnetization typical of a canted antiferromagnet (Fig. 2). Spontaneous magnetization of the compound (as defined from the linear extrapolation of the high field magnetization to $H=0$ ) achieves $0.35 \mathrm{emu} / \mathrm{g}$. This value is very close to those reported for the $\mathrm{Ti}^{4+}$ and $\mathrm{Nb}^{5+}$-doped $\mathrm{BiFeO}_{3}$-based perovskites, in which the formation of weak ferromagnetic state has been interpreted as associated with 
instability of the cycloidal structure with respect to lattice defects introduced by the aliovalent substitution [21-23]. It is important to note that the $x=0.1, x=0.15$, and $x=0.2$ samples demonstrate a very similar behavior of the magnetization in high magnetic fields (Fig. 2), thus assuming that the magnetic fieldstabilized and the spontaneously-stabilized weak ferromagnetic phases should have the same magnetic structure.

As was mentioned above, the appearance of spontaneous magnetization in donor or acceptordoped bismuth ferrites correlates with the microstructural changes originating from the doping-induced formation of crystal lattice defects [21-24]. It is suggested that the defects-produced local strain heterogeneities should act to suppress the cycloidal order [32]. To exclude the possibility that the same mechanism could be responsible for the existence of weak ferromagnetism in the charge-compensated $\mathrm{Bi}_{1-\mathrm{x}} \mathrm{Ca}_{\mathrm{x}} \mathrm{Fe}_{1-\mathrm{x}} \mathrm{Ti}_{\mathrm{x}} \mathrm{O}_{3}$, scanning probe microscopy measurements of the $x \leq 0.2$ compounds have been carried out. Typical images of the mechanically-polished ceramic surfaces recorded in PFM mode are shown in Fig. 3. Even though we can see that the $x=0.2$ sample exhibits a higher density of domain walls pinned by the structural imperfections [22, 23], its microstructure and ferroelectric/ferroelastic domain structure remain very similar to those specific to the antiferromagnetic compounds of $\mathrm{BiFeO}_{3}$-based perovskites $[23,33]$. In other words, the doping-driven AFM-wFM transition in the $\mathrm{Bi}_{1-\mathrm{x}} \mathrm{Ca}_{\mathrm{x}} \mathrm{Fe}_{1-\mathrm{x}} \mathrm{Ti}_{\mathrm{x}} \mathrm{O}_{3}$ system is not accompanied by any dramatic morphological changes [21-24] that might be suggestive of a coupling between the defect and magnetic structures. Accordingly, the compositional evolution of magnetic properties of the $\mathrm{Bi}_{1-x} \mathrm{Ca}_{x} \mathrm{Fe}_{1-\mathrm{x}} \mathrm{Ti}_{\mathrm{x}} \mathrm{O}_{3}$ samples should be mainly determined by the cooperative structural distortions introduced by the chemical replacement (the variation in the critical fields stabilizing the canted magnetic structure in $\mathrm{BiFeO}_{3}$-based multiferroics reflects the substitution-driven changes in the magnetocrystalline anisotropy) [7-9]. In contrast to the $\mathrm{Bi}_{1-x} L_{x} \mathrm{FeO}_{3}$ series [8-12], where the establishment of a weak ferromagnetic state is accompanied by the destruction of the initial polar structure, the $\mathrm{Bi}_{1-\mathrm{x}} \mathrm{Ca}_{\mathrm{x}} \mathrm{Fe}_{1-\mathrm{x}} \mathrm{Ti}_{\mathrm{x}} \mathrm{O}_{3}$ system provides a very uncommon possibility to observe the chemical pressure-induced spontaneous magnetization in the ferroelectric phase. Recognition of the lattice-related factors controlling the magnetic and structural instabilities in $\mathrm{BiFeO}_{3}$ requires a systematic investigation of the $B i_{1-x}^{3+} A_{x}^{2+} \mathrm{Fe}_{1-x}^{3+} B_{x}^{4+} \mathrm{O}_{3}^{2-}$ solid solutions that will be the objective of our future research.

In conclusion, the study of crystal structure as well as magnetic and local ferroelectric properties of the $\mathrm{Bi}_{1-x} \mathrm{Ca}_{x} \mathrm{Fe}_{1-\mathrm{x}} \mathrm{Ti}_{\mathrm{x}} \mathrm{O}_{3}(x \leq 0.2)$ compounds has been performed to revise some previously published data [25-27] and determine whether the $\mathrm{Ca}^{2+} / \mathrm{Ti}^{4+}$ co-substitution can act to stabilize a weak ferromagnetic/ferroelectric state. The investigation, for the first time, has allowed to establish the exact 
compositional boundary $(x=0.15)$ separating the antiferromagnetic and weak ferromagnetic phases and describe the behavior of the cycloidal structure under external magnetic field. It has been shown that the doping-induced AFM-wFM transformation is associated neither with changes in the symmetry of the crystal lattice nor with the local structural defects. The results suggest the possibility of an alternative approach to tailoring the multiferroic properties of $\mathrm{BiFeO}_{3}$-based perovskites connected with the control of the magnetic anisotropy via a doping-introduced chemical pressure.

\section{Acknowledgements}

This work was supported by funds from FEDER (Programa Operacional Factores de Competitividade COMPETE) and from FCT-Fundação para a Ciência e a Tecnologia under the project PEst-C/FIS/UI0036/2014. V.A.K. is grateful to Fundação para a Ciência e a Tecnologia for financial support through the FCT Investigator Programme (project IF/00819/2014). Access to TAIL-UC facility funded under QREN-Mais Centro project ICT_2009_02_012_1890 is gratefully acknowledged.

\section{References}

1. S. Fusil, V. Garcia, A. Barthélémy, and M. Bibes, Annu. Rev. Mater. Res. 44 (2014) 91-116.

2. D. Sando, A. Barthélémy, and M. Bibes, J. Phys.: Condens. Matter 26 (2014) 473201.

3. F. Kubel, H. Schmid, Acta Cryst. B 46 (1990) 698-702.

4. S. V. Kiselev, R. P. Ozerov, and G. S. Zhdanov, Sov. Phys.-Dokl. 7 (1963) 742.

5. C. Ederer and N. A. Spaldin, Phys. Rev. B 71 (2005) 060401.

6. R. D. Johnson, P. Barone, A. Bombardi, R. J. Bean, S. Picozzi, P. G. Radaelli, Y. S. Oh, S.-W. Cheong, and L. C. Chapon, Phys. Rev. Lett. 110 (2013) 217206.

7. A. M. Kadomtseva, Yu. F. Popov, A. P. Pyatakov, G. P. Vorob'ev, A. K. Zvezdin, and D. Viehland, Phase Transit. 79 (2006) 1019-1042.

8. G. Le Bras, D. Colson, A. Forget, N. Genand-Riondet, R. Tourbot, and P. Bonville, Phys. Rev. B 80 (2009) 134417.

9. P. Chen, Ö. Günaydın-Şen, W. J. Ren, Z. Qin, T. V. Brinzari, S. McGill, S.-W. Cheong, and J. L. Musfeldt, Phys. Rev. B 86 (2012) 014407.

10. I. O. Troyanchuk, D. V. Karpinsky, M. V. Bushinsky, V. A. Khomchenko, G. N. Kakazei, J. P. Araujo, M. Tovar, V. Sikolenko, V. Efimov, and A. L. Kholkin, Phys. Rev. B 83 (2011) 054109. 
11. V. A. Khomchenko, L. C. J. Pereira, and J. A. Paixão, J. Phys. D: Appl. Phys. 44 (2011) 185406.

12. V. A. Khomchenko, I. O. Troyanchuk, D. V. Karpinsky, and J. A. Paixão, J. Mater. Sci. 47 (2012) 1578-1581.

13. T. D. Rao, A. Kumari, M. K. Niranjan, and S. Asthana, Physica B 448 (2014) 267-272.

14. C. A. Wang, H. Z. Pang, A. H. Zhang, X. B. Lu, X. S. Gao, M. Zeng, and J.-M. Liu, Mater. Res. Bull. 70 (2015) 595-599.

15. I. O. Troyanchuk, A. N. Chobot, O. S. Mantytskaya, and N. V. Tereshko, Inorg. Mater. 46 (2010) 424-428.

16. A. Ianculescu, F. P. Gheorghiu, P. Postolache, O. Oprea, and L. Mitoseriu, J. Alloy. Compd. 504 (2010) 420-426.

17. V. A. Khomchenko, I. O. Troyanchuk, D. M. Többens, V. Sikolenko, and J. A. Paixão, J. Phys.: Condens. Matter 25 (2013) 135902.

18. I. O. Troyanchuk, M. V. Bushinsky, D. V. Karpinsky, V. Sirenko, V. Sikolenko, and V. Efimov, Eur. Phys. J. B 73 (2010) 375-381.

19. V. A. Khomchenko, I. O. Troyanchuk, V. Sikolenko, and J. A. Paixão, J. Mater. Sci. 48 (2013) 38523856.

20. P. C. Sati, M. Arora, S. Chauhan, M. Kumar, and S. Chhoker, J. Mater. Sci.: Mater. Electron. 24 (2013) 5023-5034.

21. V. A. Khomchenko and J. A. Paixão, Mater. Lett. 169 (2016) 180-184.

22. V. A. Khomchenko and J. A. Paixão, J. Phys. D: Appl. Phys. 48 (2015) 345001.

23. V. A. Khomchenko and J. A. Paixão, J. Mater. Sci. 50 (2015) 7192-7196.

24. V. A. Khomchenko and J. A. Paixão, J. Appl. Phys. 116 (2014) 214105.

25. D. V. Karpinsky, I. O. Troyanchuk, J. V. Vidal, N. A. Sobolev, and A. L. Kholkin, Solid State Commun. 151 (2011) 536-540.

26. Q. Q. Wang, Z. Wang, X. Q. Liu, and X. M. Chen, J. Am. Ceram. Soc. 95 (2012) 670-675.

27. P. Kumar and M. Kar, J. Alloy. Compd. 584 (2014) 566-572.

28. A. Le Bail, H. Duroy, and J. L. Fourquet, Mat. Res. Bull. 23 (1988) 447-452.

29. J. Rodríguez-Carvajal, Physica B 192 (1993) 55-69.

30. R. D. Shannon, Acta Cryst. A 32 (1976) 751-767.

31. V. A. Khomchenko, L. C. J. Pereira, and J. A. Paixão, J. Appl. Phys. 115 (2014) 164101.

32. J. A. Schiemer, R. L. Withers, Y. Liu, and M. A. Carpenter, Chem. Mater. 25 (2013) 4436-4446.

33. V. A. Khomchenko, L. C. J. Pereira, and J. A. Paixão, J. Appl. Phys. 115 (2014) 034102. 
Fig. 1 Observed, calculated and difference XRD patterns obtained for the $\mathrm{Bi}_{0.9} \mathrm{Ca}_{0.1} \mathrm{Fe}_{0.9} \mathrm{Ti}_{0.1} \mathrm{O}_{3}$ compound at room temperature. The positions of the allowed Bragg reflections are marked by ticks (the low intensity peaks seen at around $20^{\circ}$ and $29^{\circ}$ are due to the small $K_{\beta}$ component of the radiation leaking through the $K_{\beta}$ filter). The central inset showing the change in the position of the (024) peak (the intensities are normalized; the $K_{\alpha 1}$ and $K_{\alpha 2}$ components of the peak are seen) illustrates the effect of Ca/Ti substitution on the crystal lattice. The right inset demonstrates normalized lattice parameters of the $\mathrm{Bi}_{1-\mathrm{x}} \mathrm{Ca}_{\mathrm{x}} \mathrm{Fe}_{1-\mathrm{x}} \mathrm{Ti}_{\mathrm{x}} \mathrm{O}_{3}$ compounds as a function of $\mathrm{Ca} / \mathrm{Ti}$ content.

Fig. 2 Magnetic hysteresis loops of the $\mathrm{Bi}_{1-\mathrm{x}} \mathrm{Ca}_{\mathrm{x}} \mathrm{Fe}_{1-\mathrm{x}} \mathrm{Ti}_{\mathrm{x}} \mathrm{O}_{3}$ compounds at room temperature.

Fig. 3 Vertical PFM images $(10 \times 5 \mu \mathrm{m})$ of the mechanically-polished ceramics of $\mathrm{Bi}_{1-\mathrm{x}} \mathrm{Ca}_{\mathrm{x}} \mathrm{Fe}_{1-\mathrm{x}} \mathrm{Ti}_{\mathrm{x}} \mathrm{O}_{3}$.

Domains with different orientation of polarization are distinguished by different contrast.

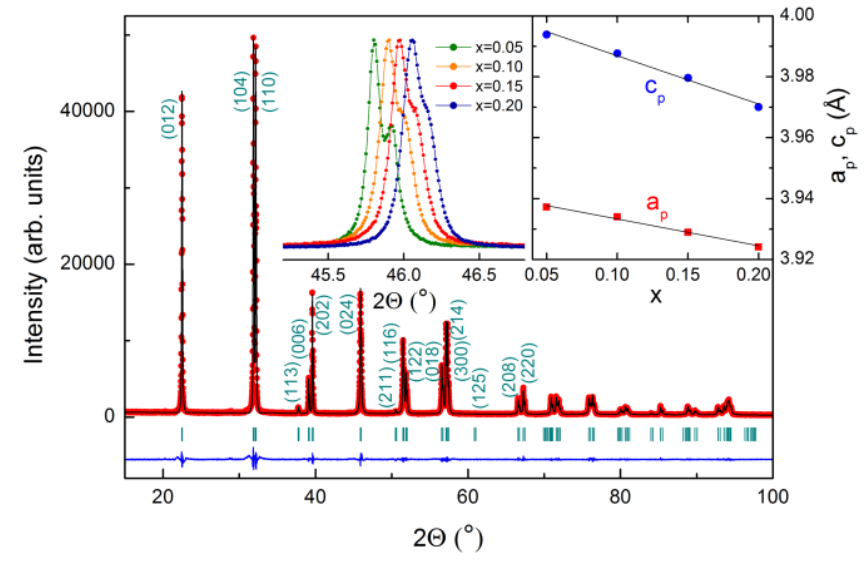

Figure 1

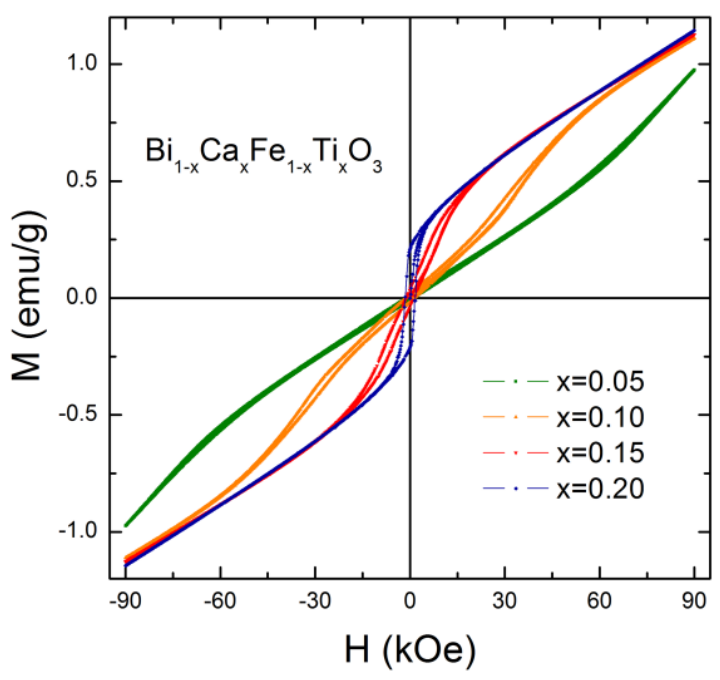

Figure 2
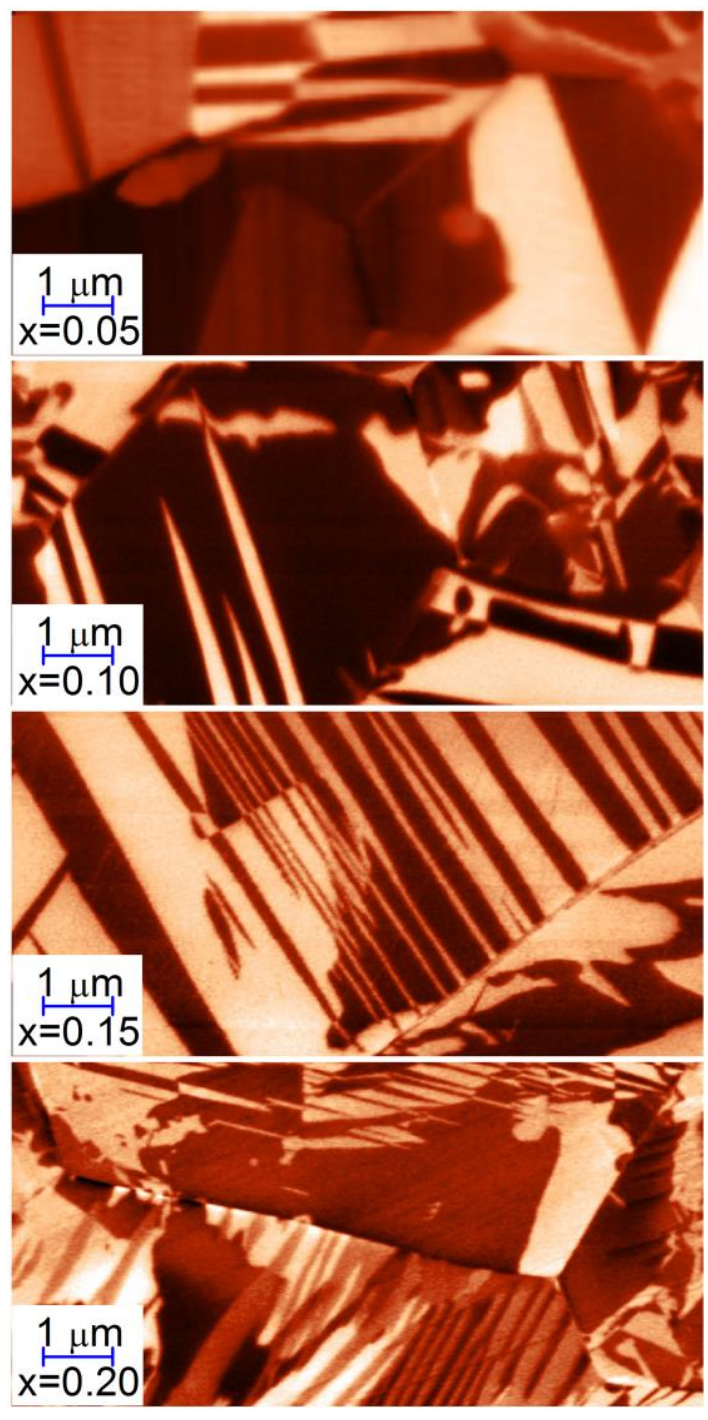

Figure 3 
